\title{
Incidência e fatores associados ao desmame simples, difícil e prolongado em uma unidade de terapia intensiva
}

\author{
Incidence and associated factors to simple, difficult and prolonged weaning \\ in an intensive care unit
}

\author{
Paula Caitano Fontela ${ }^{a}$, Heloísa Meincke Eickhoff ${ }^{b}$, Eliane Roseli Winkelmannc \\ a Fisioterapeuta. Mestranda do Programa de Pós-Graduação em Ciências Pneumológicas da Universidade Federal do Rio Grande do Sul (UFRGS), Porto Alegre, RS, Brasil. \\ b Fisioterapeuta. Mestre em Educação nas Ciências pela Universidade Regional do Noroeste do Estado do Rio Grande do Sul (UNIIJUÍ). Docente do Departamento de \\ Ciências da Vida da UNIIJUÍ, Ijuí, RS, Brasil. \\ Fisioterapeuta. Doutora em Ciências da Saúde pela UFRGS. Docente do Programa de Pós-Graduação Stricto Sensu Mestrado em Atenção Integral à Saúde - PPGAIS e \\ do Departamento de Ciências da Vida da UNIIJUÍ, Ijuí, RS, Brasil.
}

RESUMO

Introdução: A classificação do desmame ventilatório é uma ferramenta útil e importante dentro das unidades de terapia intensiva (UTI) com o intuito de conhecer a realidade do serviço.

Objetivo: Determinar a incidência e os fatores associados ao desmame simples, difícil e prolongado em pacientes internados em uma UTI geral adulto.

Materiais e Métodos: Estudo de coorte prospectivo realizado em um Hospital de Grande Porte no interior do Estado do Rio Grande do Sul - Brasil, entre janeiro e abril de 2014. Foram incluídos 25 pacientes submetidos à ventilação mecânica invasiva (VM) em tempo superior a 24 horas e que realizaram o teste de respiração espontânea.

Resultados: A incidência de desmame simples, difícil e prolongado foi de $52 \%(n=13), 32 \%(n=8)$ e $16 \%(n=4)$, respectivamente. Os dias de internação na UTI $(p=0,039)$, dias de VM $(p=0,002)$ e dias de desmame da VM $(p<0,001)$ foram superiores no grupo desmame prolongado. A incidência de pneumonia associada à VM (PAV) $(p=0,016)$, realização de traqueostomia $(p=0,045)$, VM prolongada $(p=0,011)$ e VM controlada prolongada $(p=0,028)$ foi maior no grupo desmame prolongado. Houve uma tendência de maior mortalidade na UTI no grupo desmame prolongado, no entanto, sem diferença estatisticamente significativa $(p=0,066)$.

Conclusão: O desmame prolongado está associado ao aumento nos dias de internação na UTI, dias de VM e dias de desmame da VM, assim como na incidência de PAV, realização de traqueostomia, VM prolongada e VM controlada prolongada. Esses resultados nos mostram que nenhum esforço deve ser poupado em relação ao desmame, devendo esse ser otimizado a fim de evitar ou pelo menos reduzir complicações e intervenções.

Palavras-chave: epidemiologia; desmame do respirador; classificação; unidades de terapia intensiva.

Introduction: The weaning classification is a useful and important tool in the intensive care units (ICUs) in order to know the reality of the service.

Objective: To determine the incidence of simple, difficult and prolonged weaning and its associated factors in patients admitted in a general ICU for adults.

Materials and Methods: A prospective cohort study was carried out in a great hospital in the countryside of Rio Grande do Sul state, Brazil, from January to April, 2014. We included 25 patients submitted to invasive mechanical ventilation (MV) for more than 24 hours and that performed the spontaneous breathing test.

Results: The incidence of simple, difficult and prolonged weaning were $52 \%(n=13), 32 \%(n=8)$ and $16 \%(n=4)$, respectively. The days in the ICU $(p=0.039)$, days of MV $(p=0.002)$, and days of weaning $(p<0.001)$ were higher in the prolonged weaning group. The incidence of pneumonia associated to $M V(p=0.016)$, tracheostomy $(p=0.045)$, prolonged $M V(p=0.011)$, and prolonged controlled MV $(p=0.028)$ was higher in the prolonged weaning group. There was a trend toward higher mortality in the ICU in the prolonged weaning group, although without statistically significant difference $(p=0.066)$.

Conclusion: Prolonged weaning is associated with increased days in the ICU, days of MV, and days of ventilator weaning, as well as the incidence of ventilator-associated pneumonia, tracheostomy, prolonged MV, and prolonged controlled MV. The results show that no efforts should be spared regarding weaning and it should be optimized to avoid or at least reduce complications and interventions.

Keywords: epidemiology; ventilator weaning; classification; intensive care units. 


\section{INTRODUÇÃO}

O desmame é definido como o processo de transição da ventilação artificial para a espontânea nos pacientes que permanecem em ventilação mecânica invasiva (VM) por tempo superior a 24 horas $^{1,2}$. Aproximadamente $40 \%$ da duração total em que o paciente permanece em VM é ocupado na tentativa de retirá-lo do aparelho ventilatório ${ }^{3,4}$. Nesse contexto, o desmame ventilatório é um processo importante dentro das unidades de terapia intensiva (UTI) cujo início jamais deve ser prorrogado, levando em consideração as complicações associadas à VM, os recursos e os custos relacionados com esse processo. O desmame da VM é geralmente bem sucedido para a maioria dos pacientes ${ }^{5}$, embora, para $20 \%$ desses, haja falhas na primeira tentativa do teste de respiração espontânea (TRE) .

A VM prolongada está associada a várias complicações, como pneumonia associada à $\mathrm{VM}^{6}$, disfunção diafragmática induzida pela $\mathrm{VM}^{7,8}$, polineuropatia do doente $\mathrm{Crítico}^{9}$, entre outras. As principais causas de dependência da VM são causas neurológicas ${ }^{10,11}$, por comprometimentos do sistema respiratório ou do sistema cardiovascular ${ }^{10,11,12,13}$, e fatores psicológicos ${ }^{10,11}$. Distúrbios metabólicos, endócrinos, e eletrolíticos, bem como desnutrição, obesidade e anemia também podem contribuir para o tempo de $\mathrm{VM}^{10}$.

Atualmente, o TRE é o principal teste diagnóstico para determinar se os pacientes podem ser extubados com sucesso $^{10}$. A classificação do desmame ventilatório torna-se uma ferramenta útil e importante dentro das UTIs com o intuito de conhecer a realidade do serviço, e dessa forma, qualificar as ações desenvolvidas, visando otimizar o desmame da VM. A fisioterapia tem papel fundamental na assistência do desmame da VM. Estudos ${ }^{14,15}$ demonstram que, a condução de protocolos de triagem para a interrupção da VM realizados por fisioterapeutas, reduz o tempo de VM e a morbidade ${ }^{14}$ e aumenta a taxa de sucesso no desmame ${ }^{15}$.

O conhecimento dos fatores e métodos que potencializem ao máximo à possibilidade de descontinuação da VM e o entendimento da realidade do serviço contribuem no processo de desmame e consequentemente na evolução e no prognóstico de vida dos pacientes críticos. Portanto, o objetivo deste estudo foi determinar a incidência e os fatores associados ao desmame simples, difícil e prolongado em pacientes internados em uma UTI geral adulto.

\section{MATERIAIS E MÉTODOS}

Trata-se de um estudo de coorte prospectivo realizado por meio da coleta de dados registrados em prontuários de pacientes sob VM invasiva internados na UTI geral adulto de um Hospital de Grande Porte no interior do Estado do
Rio Grande do Sul, composta por 10 leitos ocupados por pacientes clínicos e cirúrgicos. A pesquisa ocorreu no período entre janeiro e abril de 2014. O estudo foi desenvolvido após aprovação da Comissão de Avaliação de Trabalhos Acadêmicos do referido hospital e do Comitê de Ética em Pesquisa da Universidade Regional do Noroeste do Estado do Rio Grande do Sul (no 483.508/2013).

Foram incluídos, neste estudo, todos os pacientes submetidos à VM invasiva com duração mínima de 24 horas e que realizaram o TRE. O TRE foi realizado de acordo com critérios estabelecidos pelo próprio profissional, visto que não está implementado nesta UTI um protocolo padronizado para triagem dos critérios de elegibilidade para o TRE. Os critérios de exclusão foram pacientes menores de 18 anos, pacientes com auto-extubação, reintubação para substituição de um tubo endotraqueal com defeito, mulheres em período gestacional, pacientes que foram a óbito ou transferidos antes do término do desmame.

Para caracterizar a amostra, foram registrados a idade e o sexo dos pacientes; o diagnóstico médico principal no momento da admissão na UTI, o qual foi classificado em categorias conforme o sistema acometido (neurológico, respiratório, renal, cardiovascular, digestivo e hematopoiético); o momento em que foi instituída a VM (na admissão ou durante a internação na UTI), causa da intubação e o início da VM.

Além disso, os pacientes foram classificados como sucesso ou falha de extubação, sendo considerado falha da extubação a necessidade de retorno à VM invasiva até $48 \mathrm{~h}$ após a retirada da prótese ventilatória ${ }^{1}$. Os pacientes que foram submetidos à traqueostomia, antes de serem desmamados, foram considerados extubados com sucesso quando estavam respirando espontaneamente através da cânula traqueal ou diretamente através do traqueostomia por 48 horas sem qualquer suporte ${ }^{10,16}$.

Se o paciente foi reintubado após o desmame primário com sucesso e posteriormente recomeçado o desmame secundário, apenas o desmame secundário foi usado para definir a classificação do desmame do paciente. A duração total do desmame foi determinada para os pacientes que foram desmamados com sucesso, sendo calculada como os dias entre o momento em que o paciente realizou o primeiro TRE até o momento em que o paciente foi desmamado com sucesso pela última vez.

Com base na duração total do desmame, bem como no número de tentativas de respiração espontânea necessários para liberar o paciente do ventilador, o desmame foi categorizado em três grupos: simples, difícil e prolongado. O desmame simples é definido como aquele em que o paciente é extubado com sucesso após o primeiro TRE ${ }^{10}$. 
Desmame difícil é aquele no qual há falha na tentativa inicial, o paciente é extubado após dois ou três TRE ou até sete dias após a primeira tentativa ${ }^{10}$. No desmame prolongado, há falha em ao menos três testes e requer mais de sete dias após o primeiro TRE para que o paciente seja desmamado com sucesso ${ }^{10}$. Esta classificação também está orientada nas últimas Diretrizes Brasileiras de $\mathrm{VM}^{16}$.

Definiu-se VM prolongada a dependência da assistência ventilatória invasiva por tempo superior a três semanas, apesar de programas de reabilitação, correção de distúrbios funcionais e utilização de novas técnicas de ventilação A VM prolongada foi analisada como variável quantitativa (dicotomizada tendo como ponto de corte 21 dias). A VM controlada prolongada (dicotomizada tendo como ponto de corte 7 dias). Outras variáveis analisadas foram dicotomizadas em sim ou não, a saber: traqueostomia e mortalidade.

Caracterizou-se com pneumonia associada à ventilação mecânica (PAV), o paciente com quadro sugestivo recente de infecção respiratória, com no mínimo 3 entre os 5 critérios: secreção traqueal purulenta/amarela; infiltrado pulmonar radiológico; leucocitose ou leucopenia; febre; e piora funcional pulmonar (diminuição da $\mathrm{PaO}_{2} / \mathrm{FiO}_{2}$ ), sendo que deveria haver pelo menos um dos dois primeiros critérios $^{17}$.

A amostra deste estudo foi resultado da inclusão dos pacientes que necessitaram de VM por tempo superior a 24 horas, internados no período referente a três meses, não sendo realizado cálculo amostral. Todos os pacientes foram acompanhados até a alta da UTI ou óbito. Considerou-se como variável dependente as novas categorias do desmame ventilatório ${ }^{10}$, constituindo-se de três grupos: simples, difícil e prolongado.
Para análise estatística, utilizou-se o software Statistical Package for the Social Science (SPSS), versão 18.0. Foi utilizado o teste qui-quadrado de Pearson para comparar a tendência das variáveis categóricas entre as três categorias do desmame. Utilizou-se o teste one-way ANOVA para variáveis contínuas com distribuição normal e teste $\mathrm{H}$ de Kruskal-Wallis para variáveis contínuas sem distribuição normal. Quando os grupos eram estatisticamente diferentes para uma variável, cada grupo foi comparado com outro através da análise post-hoc realizada pelo teste de Tukey. As variáveis categóricas foram expressas em porcentagens, e as variáveis contínuas em média \pm desvio padrão ou mediana (valor mínimo e máximo), conforme o caso. Considerou-se estatisticamente significativo os valores com $\mathrm{p}<0,05$.

\section{RESULTADOS}

Durante o período do estudo, foram admitidos 135 pacientes na UTI. Destes, 39 pacientes permaneceram em VM por mais de 24 horas e realizaram o TRE. Seis pacientes foram excluídos (um por ser menor de 18 anos, um devido auto-extubação, três por reintubação para substituição de um tubo orotraqueal com defeito e um devido transferência para outro hospital). Oito pacientes foram a óbito durante o desmame, portanto, incluiu-se 25 pacientes na coorte final. A incidência de desmame simples, difícil e prolongado foi de 52, 32 e 16\%, respectivamente (Figura 1).

A Tabela 1 mostra os dados demográficos, os diagnósticos clínicos categorizados por sistemas e as causas para o início da VM. A mediana de idade dos pacientes foi de 58 anos, com idade mínima de 20 e máxima de 84 anos.

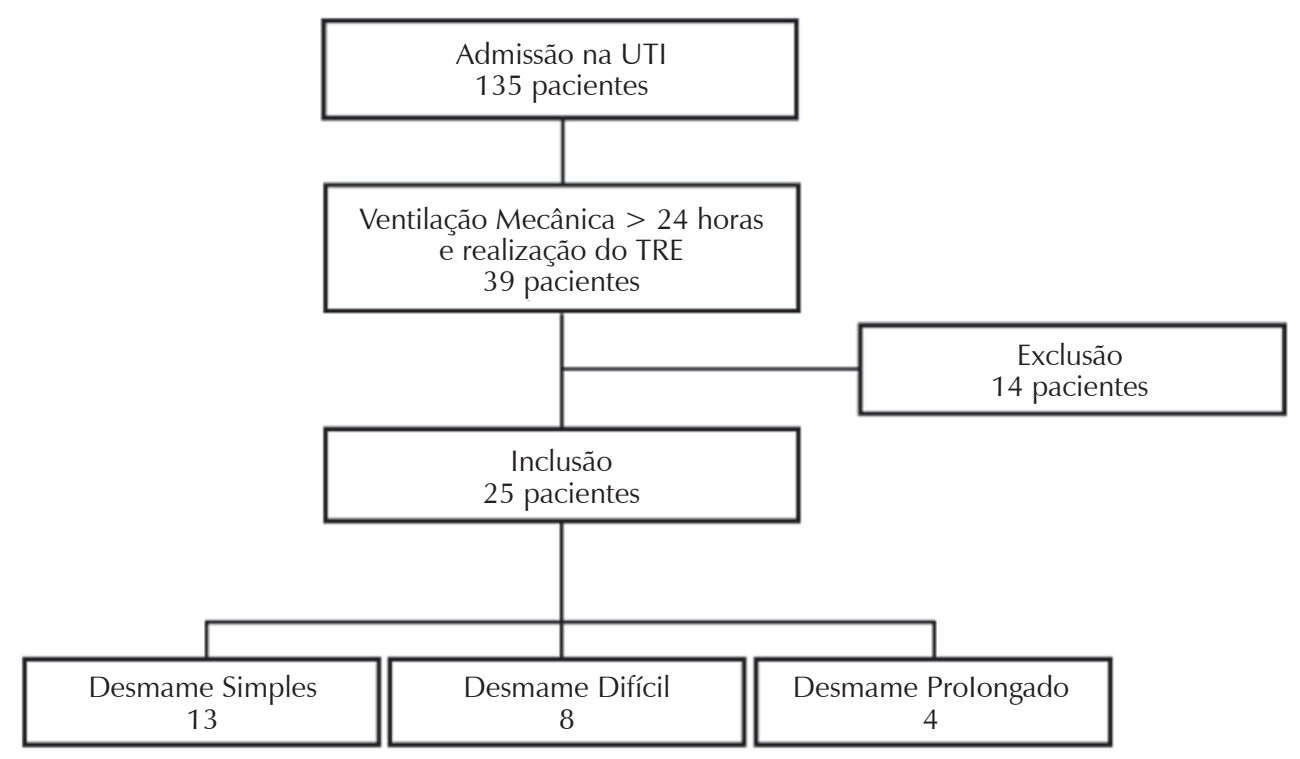

Figura 1. Fluxograma do estudo. UTI: unidade de terapia intensiva; TRE: teste de respiração espontânea. 
Tabela 1. Caracterização dos pacientes de acordo com variáveis demográficas e clínicas.

\begin{tabular}{lc}
\hline \multicolumn{1}{c}{ Variáveis } & $\mathbf{n}=\mathbf{2 5}$ \\
Sexo & $18(72,0)$ \\
Masculino & $59,0 \pm 17,3$ \\
Idade (anos completos) & \\
Diagnóstico clínico principal & $8(32,0)$ \\
Neurológico & $6(24,0)$ \\
Respiratório & $4(16,0)$ \\
Renal & $3(12,0)$ \\
Cardiovascular & $3(12,0)$ \\
Digestivo & $1(4,0)$ \\
Hematopoético & \\
Necessidade de VM & $23(92,0)$ \\
Na admissão na UTI & $2(8,0)$ \\
Durante a internação & \\
Causa intubação e início VM & $2(8,0)$ \\
Hipoxemia & $6(24,0)$ \\
Aumento do trabalho respiratório & $14(56,0)$ \\
Diminuição do nível de consciência & $3(12,0)$ \\
PCR &
\end{tabular}

VM: ventilação mecânica; UTI: unidade de terapia intensiva;

PCR: parada cardiorrespiratória.

Resultados expressos por número (\%) ou média \pm desvio padrão.
Os dias de internação na UTI, dias de VM e dias de desmame ventilatório diferiram entre os grupos de desmame (Tabela 2). O grupo desmame prolongado apresentou aumento significativo nos dias de internação na UTI em relação ao grupo desmame simples, mas não em relação ao grupo desmame difícil. Na variável dias de VM, o grupo desmame prolongado apresentou aumento significativo em relação ao grupo desmame simples e difícil, e nos dias de desmame da VM houve aumento estatisticamente significativo no grupo desmame prolongado em relação aos grupos desmame simples e difícil. Em nenhuma das variáveis houve diferença significativa entre os grupos desmame simples e difícil $(p>0,05)$.

A incidência de PAV, realização de traqueostomia, VM prolongada e VM controlada prolongada foi maior no grupo desmame prolongado (Tabela 2).

\section{DISCUSSÃO}

Em 2005, a Conferência Internacional sobre Desmame da Ventilação Mecânica ${ }^{10}$, propôs um novo sistema de classificação baseado na dificuldade e duração do desmame. No presente estudo, a aplicação desse sistema de classificação em pacientes ventilados por mais de 24 horas encontrou diferenças significativas nos dias de internação na UTI, dias de VM, dias de desmame da VM, incidência de PAV,

Tabela 2. Comparação de características demográficas, clínicas e epidemiológicas baseada nas novas categorias do desmame ventilatório.

\begin{tabular}{|c|c|c|c|c|}
\hline \multirow{2}{*}{ Variáveis } & \multicolumn{3}{|c|}{ Tipo desmame } & \multirow{2}{*}{ Valor de $\mathrm{p}$} \\
\hline & Simples $(n=13)$ & Difícil $(n=8)$ & Prolongado $(n=4)$ & \\
\hline Sexo Masculino & $13(100)$ & $4(50,0)$ & $1(25,0)$ & $0,001 *$ \\
\hline Idade (anos) & $55,1 \pm 16,1$ & $59,3 \pm 18,1$ & $71,5 \pm 17,7$ & $0,261^{\epsilon}$ \\
\hline Dias de internação na UTI & $14,3 \pm 9,8^{b}$ & $14,0 \pm 7,0^{\mathrm{a}}$ & $28,3 \pm 12,1^{\text {a }}$ & $0,039^{\epsilon *}$ \\
\hline Dias de sedação & $6,2 \pm 7,6$ & $4,6 \pm 2,1$ & $6,7 \pm 4,1$ & $0,798^{\epsilon}$ \\
\hline Dias de VM & $8,7 \pm 6,8^{b}$ & $11,9 \pm 6,1^{b}$ & $27,0 \pm 13,6^{a}$ & $0,002^{\epsilon *}$ \\
\hline Dias de VM controlada & $6,6 \pm 7,5$ & $6,1 \pm 2,4$ & $9,5 \pm 5,8$ & $0,645^{£}$ \\
\hline Dias de desmame da VM & $3,1 \pm 1,3^{b}$ & $6,7 \pm 3,9^{b}$ & $18,3 \pm 7,9^{a}$ & $<0,001^{\epsilon *}$ \\
\hline PAV & $3(23,1)$ & $0(0,0)$ & $3(75,0)$ & $0,016^{*}$ \\
\hline Traqueostomia & $6(46,2)$ & $7(87,5)$ & $4(100)$ & $0,045^{*}$ \\
\hline VM prolongada ( $\geq 21$ dias) & $1(7,7)$ & $1(12,5)$ & $3(75,0)$ & $0,011^{*}$ \\
\hline VM controlada prolongada ( $\geq 7$ dias) & $1(7,7)$ & $2(25,0)$ & $3(75,0)$ & $0,028^{*}$ \\
\hline Sucesso no desmame $\neq$ & $12(92,3)$ & $6(75,0)$ & $1(25,0)$ & 0,812 \\
\hline Mortalidade na UTI & $2(15,4)$ & $2(25,0)$ & $3(75,0)$ & 0,066 \\
\hline
\end{tabular}

UTI: unidade de terapia intensiva; VM: ventilação mecânica; PAV: pneumonia associada a ventilação mecânica.

Resultados expressos em média \pm desvio padrão e mediana (valor mínimo-valor máximo).

F Variável analisada em 21 pacientes, visto que 4 pacientes foram a óbito antes de completar 48 horas após a extubação, sendo 1 paciente do grupo desmame difícil e

3 do grupo desmame prolongado.

$€$ Teste one-way ANOVA.

$£$ Teste $\mathrm{H}$ de Kruskal-Wallis; Teste do qui-quadrado de Pearson para variáveis qualitativas.

* Resultado significativo: $\mathrm{p} \leq 0,05$.

Letras distintas entre o grupo desmame prolongado e os grupos desmame simples e difícil representam médias significativamente diferentes $(p<0,05)$. 
traqueostomia, VM prolongada e VM controlada prolongada, confirmando assim a relevância clínica do novo sistema de classificação. Entretanto, a classificação do desmame não impactou na incidência de mortalidade na UTI.

O primeiro estudo de coorte prospectivo que aplicou as novas categorias para o desmame da VM foi desenvolvido por Funk et al. ${ }^{18}$. Estes pesquisadores realizaram o estudo em cinco UTIs de quatro hospitais municipais de Viena na Áustria e avaliaram 257 pacientes em desmame. As proporções encontradas de acordo com as novas categorias foram: 59,1, 26,5 e 14,4\% para desmame simples, difícil e prolongado, respectivamente. Estas proporções são comparáveis com as da presente pesquisa, levando em consideração que os pacientes do estudo de Funk et al. ${ }^{18}$ eram mais jovens e tinham grande prevalência de internações cirúrgicas, além disso, os autores incluíram na investigação todos os pacientes, inclusive aqueles que necessitaram de VM por menos de 24 horas. Entretanto, o desmame só é considerado em pacientes que permanecem em VM invasiva por tempo superior a 24 horas $^{1,2}$, o que justifica os critérios de inclusão do presente estudo.

A maioria dos estudos ${ }^{19,20,21}$ realizados para estimar a incidência de cada grupo de desmame avalia pacientes ventilados por mais de 48 horas $^{19,20}$ ou superior a 24 horas $^{21}$. A principal diferença é observada na proporção de pacientes no grupo desmame simples que é provavelmente relacionada com o desenho do protocolo de desmame e/ou com os critérios de inclusão dos estudos. Nesses estudos, fatores preditivos de desmame foram usados como critérios para a realização do TRE. O desmame só foi iniciado quando o índice de respiração rápida e superficial não excedeu 105 ciclos/min/ $\mathrm{L}^{19}$, ou se após 3-5 minutos de ventilação espontânea o paciente tinha pelo menos dois dos seguintes critérios: frequência respiratória inferior a 35 respiração/ minuto, pressão inspiratória máxima abaixo de $-20 \mathrm{cmH}_{2} \mathrm{O}$ ou volume corrente acima de $5 \mathrm{~mL} / \mathrm{kg}$ do peso predito ${ }^{21}$. É importante salientar que na rotina da UTI, onde o presente estudo foi realizado, não estão protocolados critérios para a realização do TRE, o que pode ter influenciado nas incidências do desmame ventilatório, principalmente no grupo desmame simples como constatado anteriormente.

O estudo de Funk et al. ${ }^{18}$ revelou também que os pacientes com desmame prolongado apresentavam maior mortalidade (32\%) em comparação com aqueles de desmame difícil (9\%) e simples (13\%) ( $p=0,0205)$. No presente estudo, apesar de haver diferença em relação a incidência de mortalidade entre os grupos de desmame esta não foi estatisticamente significativa, talvez pelo pequeno número de pacientes incluídos. Em estudo realizado por Esteban et al. ${ }^{22}$, os pacientes que foram extubados com sucesso após o primeiro TRE, ou seja, desmame simples de acordo com as novas categorias, demonstraram uma mortalidade na UTI de $11 \%$. Este resultado é comparável com o do presente estudo.

Tonnelier et al. ${ }^{23}$, realizaram um estudo recente de coorte retrospectivo na França com 115 pacientes que permaneceram em VM por mais de 48 horas e que realizaram o TRE. Evidenciaram um índice de desmame simples, difícil e prolongado de 30, 40 e 30\%, respectivamente, e constataram que o desmame prolongado foi um fator independente de risco para maior mortalidade na UTI (odds ratio: 15,11; IC 95\%: 1,61-141,91; $p=0,01$ ) e mortalidade hospitalar (odds ratio: 3,66; IC 95\%: 0,9913,51; $p=0,05)$, confirmando assim a relevância clínica das novas categorias do desmame ventilatório no prognóstico dos pacientes. Entretanto, a classificação do desmame não afetou a mortalidade em um ano (odds ratio: 2,61 ; IC 95\%: $0,82-8,35 ; p=0,11)$.

A maior incidência de VM prolongada e VM controlada prolongada no grupo desmame prolongado, assim como aumento significativo no tempo de VM, dias de desmame e tempo de permanência na UTI constatadas neste estudo, podem ser explicadas pelo fato de que o paciente submetido à VM prolongada pode começar a desenvolver uma atrofia muscular respiratória por desuso, a partir de 76 horas do início da prótese ventilatória ${ }^{24}$. Isto ocorre tendo em vista que a VM não promove níveis de propriocepção que mantenha uma atividade ideal das fibras musculares ${ }^{24}$, o que explica principalmente a associação entre VM controlada prolongada e desmame prolongado, pois na ventilação controlada a atividade das fibras musculares respiratória é mínima.

Os pacientes com desmame prolongado com mais frequência foram submetidos à traqueostomia, o mesmo fato foi constado por Funk et al. ${ }^{18}$. Cabe destacar que no presente estudo uma proporção substancial de pacientes foram submetidos à traqueostomia precoce, embora não esteja definido o impacto benéfico deste procedimento. A traqueostomia precoce foi realizada em 8 pacientes, sendo que 5 destes tinham como diagnóstico clínico de admissão o neurológico, o que possivelmente levou a equipe médica a esperar por um desmame prolongado e a realizar a traqueostomia precocemente, visto que especificamente nestes pacientes, a realização precoce da traqueostomia pode ser benéfica ${ }^{25}$. Neste estudo considerou-se o ponto de corte de 8 dias para traqueostomia precoce, no entanto, as definições de traqueostomia precoce divergem muito em relação ao ponto de corte utilizado pelos estudos ${ }^{25,26}$.

A incidência de PAV foi superior no grupo de pacientes com desmame prolongado. Este mesmo achado foi encontrado por Tonnelier et al. ${ }^{23}$, os quais encontraram uma 
incidência de PAV de $62 \%$ no grupo desmame prolongado. A PAV é a infecção nosocomial mais comum no ambiente de cuidados intensivos. A prevalência é variável, com taxas desde 6 até 50 casos por 100 admissões na UTI ${ }^{27,28}$. Estudos ${ }^{27,28}$ demonstram que a incidência dessa infecção aumenta com a duração da ventilação mecânica e apontam taxas de ataque de aproximadamente $3 \%$ por dia durante os primeiros 5 dias de ventilação. O desenvolvimento de pneumonia nosocomial e no ambiente de cuidados intensivos, especificamente da $\mathrm{PAV}$, tem morbidade significativa associada, prolongando o tempo de VM, bem como o tempo de permanência na UTI, com todos os custos associados a esse prolongamento ${ }^{28,29}$.

Salienta-se que neste estudo, os pacientes foram acompanhados até a alta da UTI ou óbito. Portanto, o desmame secundário (ou seja, o desmame após a reintubação) foi considerado neste estudo, e desta forma, avaliado a verdadeira incidência de desmame prolongado e sua associação com as variáveis avaliadas no presente estudo.

O presente estudo apresenta algumas limitações, dentre elas, o curto período de acompanhamento e consequentemente seu reduzido número de pacientes incluídos no estudo, e possível fator de sazonalidade que interfira nos resultados da amostra. Além disso, a amostra provém de um único centro. Portanto, a estimativa da epidemiologia do desmame simples, difícil e prolongado provavelmente não pode ser generalizada, motivo pelo qual sugere-se que mais estudos sejam realizados para complementar tais achados. Entretanto, o presente estudo contribui com a divulgação de resultados sobre a incidência do desmame ventilatório e serve como subsídio para a realização de estudos de maior escala. Além disso, colabora para a discussão sobre a aplicabilidade e implicações geradas pelo uso desta classificação atual do desmame.

Outras limitações referem-se ao fato de que informações sobre o histórico médico dos pacientes antes da admissão na UTI não foram sistematicamente avaliadas. Portanto, a prevalência de determinadas patologias associadas e sua ausência como um fator de risco associado com a classificação de desmame pode ter sido subestimada. Além disso, não se pode fornecer informações exatas com relação aos custos do tratamento nas categorias de desmame. No entanto, o desmame prolongado foi associado com maior tempo de VM, desmame ventilatório e permanência na UTI, o que provavelmente aumenta o custo do tratamento.

Conhecer a incidência de desmame simples, difícil e prolongado da UTI revela a importância de aprimorar o processo de desmame visando um maior percentual de sucesso em menor tempo. A partir desta casuística e da realidade evidenciada, torna-se fundamental identificar demais causas de falha na retirada da VM, pois sabe-se que muitos fatores podem interferir neste processo e que estes, não foram sistematicamente avaliados no presente estudo. A identificação dos fatores associados a classificação do desmame, pode servir de base para a criação de estratégias e protocolos específicos que visem a otimização do desmame ventilatório em UTIs.

Em conclusão, neste estudo realizado com pacientes ventilados por tempo superior a 24 horas, a incidência de desmame simples, difícil e prolongado foi de 52, 32 e $16 \%$, respectivamente. O desmame prolongado está associado ao aumento nos dias de internação na UTI, de VM e de desmame ventilatório, assim como na incidência de PAV, realização de traqueostomia, VM prolongada e VM controlada prolongada. Houve uma tendência de maior mortalidade na UTI no grupo desmame prolongado. Esses resultados nos mostram que nenhum esforço deve ser poupado em relação ao desmame, devendo esse ser otimizado a fim de evitar ou pelo menos reduzir complicações e intervenções nos pacientes críticos internados em UTIs.

\section{REFERÊNCIAS}

1. Goldwasser R, Farias A, Freitas EE, Saddy F, Amado V, Okamoto VN. Desmame e interrupção da ventilação mecânica. III Consenso Brasileiro de Ventilação Mecânica. Rev Bras Ter Intensiva. 2007;13(3):384-92. http://dx.doi.org/10.1590/S0103507X2007000300021

2. Presto B, Damásio L. Fisioterapia respiratória. 4th ed. Rio de Janeiro: Elsevier; 2009.

3. Esteban A, Anzueto A, Frutos F, Alía I, Brochard L, Stewart TE, Benito S, Epstein SK, Apezteguía C, Nightingale P, Arroliga AC, Tobin MJ; Mechanical Ventilation International Study Group. Characteristics and outcomes in adult patients receiving mechanical ventilation: a 28-day international study. JAMA. 2002;287(3):345-55. http:// dx.doi.org/10.1001/jama.287.3.345

4. Ely EW, Baker AM, Dunagan DP, Burke HL, Smith AC, Kelly PT, Johnson MM, Browder RW, Bowton DL, Haponik EF. Effect on the duration of mechanical ventilation of identifying patients capable of breathing spontaneously. N Engl J Med. 1996;335(25):1864-9. http://dx.doi.org/10.1056/NEJM199612193352502

5. Eskandar N, Apostolakos MJ. Weaning from mechanical ventilation. Crit Care Clin. 2007;23(2):263-74. http://dx.doi.org/10.1016/j. cCc.2006.12.002

6. Ntoumenopoulos G, Presneill JJ, McElholum M, Cade JF. Chest physiotherapy for the prevention of ventilator-associated pneumonia. Intensive Care Med. 2002;28(7):850-6. http://dx.doi. org/10.1007/s00134-002-1342-2

7. Vassilakopoulos T, Petrof BJ. Ventilator-induced diaphragmatic dysfunction. Am J Respir Crit Care Med. 2004;169(3):336-41. http://dx.doi.org/10.1164/rccm.200304-489CP

8. Levine S, Nguyen T, Taylor N, Friscia ME, Budak MT, Rothenberg P, Zhu J, Sachdeva R, Sonnad S, Kaiser LR, Rubinstein NA, Powers SK, Shrager JB. Rapid disuse atrophy of diaphragm fibers in mechanically ventilated humans. N Engl J Med. 2008;358(13):1327-35. http:// dx.doi.org/10.1056/NEJMoa070447 
9. Frutos-Vivar F, Esteban A. Critical illness polyneuropathy: a new (or old?) reason for weaning failure. Crit Care Med. 2005;33(2):452-3. http://dx.doi.org/10.1097/01.CCM.0000153525.37281.D4

10. Boles JM, Bion J, Connors A, Herridge M, Marsh B, Melot C, Pearl R, Silverman H, Stanchina M, Vieillard-Baron A, Welte T. Weaning from mechanical ventilation. Eur Respir J. 2007;29(5):1033-56. http://dx.doi.org/10.1183/09031936.00010206

11. Maclntyre NR, Cook DJ, Ely EW Jr, Epstein SK, Fink JB, Heffner JE, Hess D, Hubmayer RD, Scheinhorn DJ; American College of Chest Physicians; American Association for Respiratory Care; American College of Critical Care Medicine. Evidence-based guidelines for weaning and discontinuing ventilatory support: a collective task force facilitated by the American College of Chest Physicians; the American Association for Respiratory Care; and the American College of Critical Care Medicine. Chest. 2001;120(6 Suppl):375S-95S. http://dx.doi.org/10.1378/chest.120.6_suppl. $375 \mathrm{~S}$

12. Jubran A, Tobin MJ. Pathophysiologic basis of acute respiratory distress in patients who fail a trial of weaning from mechanical ventilation. Am J Respir Crit Care Med. 1997;155(3):906-15. http:// dx.doi.org/10.1164/ajrccm.155.3.9117025

13. Capdevila $X$, Perrigault PF, Ramonatxo M, Roustan JP, Peray $P$, d'Athis F, Prefaut C. Changes in breathing pattern and respiratory muscle performance parameters during difficult weaning. Critical Care Med. 1998;26:79-87. http://dx.doi.org/10.1097/00003246199801000-00020

14. Ely EW, Baker AM, Dunagan DP, Burke HL, Smith AC, Kelly PT, Johnson MM, Browder RW, Bowton DL, Haponik EF. Effect on the duration of mechanical ventilation of identifying patients capable of breathing spontaneously. N Engl J Med. 1996;335(25):1864-9. http://dx.doi.org/10.1056/NEJM199612193352502

15. KollefMH, Shapiro SD, Silver P, St John RE, Prentice D, Sauer S, Ahrens TS, Shannon W, Baker-Clinkscale D. A randomized, controlled trial of protocol-directed versus physician-directed weaning from mechanical ventilation. Crit Care Med. 1997;25(4):567-74. http://dx.doi.org/10.1097/00003246-199704000-00004

16. Associação de Medicina Intensiva Brasileira; Sociedade Brasileira de Pneumologia e Tisiologia. Diretrizes Brasileiras de Ventilação Mecânica. [São Paulo]: AMIB; 2013.

17. Duarte PAD, Venazzi A, Osaku EF, Miúria CK, Schiavetto PM, Costa CRLM, Bruneri E, Eduardo AC, Vini KGP, Mora CTR, Jorge AC. Epidemiologia, estratégias e evolução de pacientes submetidos à ventilação mecânica. Rev Bras Clin Med. 2012;10(4): 302-7.

18. Funk GC, Anders S, Breyer MK, Burghuber OC, Edelmann G, Heindl W, Hinterholzer G, Kohansal R, Schuster R, SchwarzmaierD'Assie A, Valentin A, Hartl S. Incidence and outcome of weaning from mechanical ventilation according to new categories. Eur Respir J. 2010;35(1):88-94. http://dx.doi.org/10.1183/09031936. 00056909
19. Perren A, Domenighetti G, Mauri S, Genini F, Vizzardi N. Protocoldirected weaning from mechanical ventilation: clinical outcome in patients randomized for a 30-min or 120-min trial with pressure support ventilation. Intensive Care Med. 2002;28(8):1058-63. http://dx.doi.org/10.1007/s00134-002-1353-z

20. Farias JA, Retta A, Alía I, Olazarri F, Esteban A, Golubicki A, Allende D, Maliarchuk O, Peltzer C, Ratto ME, Zalazar R, Garea M,Moreno EG. A comparison of two methods to perform a breathing trial before extubation in pediatric intensive care patients. Intensive Care Med. 2001;27(10):1649-54. http://dx.doi.org/10.1007/s001340101035

21. Esteban A, Frutos F, Tobin MJ, Alía I, Solsona JF, Vallverdú I, Fernández R, de la Cal MA, Benito S, Tomás R, Spanish Lung Failure Collaborative Group. A comparison of four methods of weaning patients from mechanical ventilation. N Engl J Med. 1995;332(6):345-50. http:// dx.doi.org/10.1056/NEJM199502093320601

22. Esteban A, Alía I, Tobin MJ, Gil A, Gordo F, Vallverdú I, Blanch L, Bonet A, Vázquez A, de Pablo R, Torres A, de La Cal MA, Macías $\mathrm{S}$. Effect of spontaneous breathing trial duration on outcome of attempts to discontinue mechanical ventilation. Spanish Lung Failure Collaborative Group. Am J Respir Crit Care Med. 1999;159(2):5128. http://dx.doi.org/10.1164/ajrccm.159.2.9803106

23. Tonnelier A, Tonnelier JM, Nowak E, Gut-Gobert C, Prat G, Renault A, Boles JM, L'Her E. Clinical relevance of classification according to weaning difficulty. Respir Care. 2011;56(5):583-90. http://dx.doi. org/10.4187/respcare.00842

24. Azeredo CAC, Nemer SN, Azeredo LM. Fisioterapia respiratória em UTI. Rio de Janeiro: Lidado; 1998.

25. Pinheiro BV, Tostes RO, Brum Cl, Carvalho EV, Pinto SPS, Oliveira JCA. Traqueostomia precoce versus traqueostomia tardia em pacientes com lesão cerebral aguda grave. J Bras Pneumol. 2010;36(1):84-91. http://dx.doi.org/10.1590/S1806-37132010000100014

26. Pasini RL, Fernandes YB, Araújo S, Soares SMTP. A influência da traqueostomia precoce no desmame ventilatório de pacientes com traumatismo cranioencefálico grave. Rev Bras Ter Intensiva. 2007;19(2):176-81. http://dx.doi.org/10.1590/S0103$507 \times 2007000200006$

27. American Thoracic Society; Infectious Diseases Society of America. Guidelines for the management of adults with hospital-acquired, ventilator associated, and healthcare-associated pneumonia. Am J Respir Crit Care Med. 2005;171(4):388-416. http://dx.doi. org/10.1164/rccm.200405-644ST

28. Rello J, Diaz E. Pneumonia in the intensive care unit. Crit Care Med. 2003;31(10):2544-51. http://dx.doi.org/10.1097/01.CCM. 0000089928.84326.D2

29. Bekaert M, Timsit JF, Vansteelandt S, Depuydt P, Vésin A, Garrouste-Orgeas M, Decruyenaere J, Clec'h C, Azoulay E, Benoit D; Outcomerea Study Group. Attributable mortality of ventilatorassociated pneumonia: a reappraisal using causal analysis. Am J Respir Crit Care Med. 2011;184(10):1133-9. http://dx.doi. org/10.1164/rccm.201105-0867OC 